\title{
Characterization of Defects in Nanoparticles
}

\author{
J. Deneen and C.B. Carter
}

Department of Chemical Engineering and Materials Science, University of Minnesota, 421 Washington Ave SE, Minneapolis, MN 55455

The incorporation of nanoparticles in industrial applications requires consistent production of nanoparticles with reliable properties. Since the observed properties can strongly depend on the structure and morphology of the nanoparticles, a better understanding of the processing-structure relationship is necessary before many applications can be realized. The characterization of defects in nanoparticles can provide an explanation for their unique properties and the mechanisms responsible for particle formation and growth. This study investigates defects in various nanoparticle systems using transmission electron microscopy (TEM). Since these particles have been formed by a number of methods their chemistries and morphologies vary greatly.

The spherical nanoparticles investigated were produced by a hypersonic plasma particle deposition (HPPD) method [1]. In this process, vapor-phase precursors are injected into a flowing plasma generated by a DC arc and then rapidly quenched as they are expanded through a nozzle. The resulting nanoparticles are typically spherical and single crystal, as indicated by the concentric thickness fringes and diffraction pattern for the Si particle in Fig. 1. However, not all of the nanoparticles observed are perfect crystalline spheres, and the rare instances of defects in these particles can shed light on the formation and growth mechanisms. Occasionally a particle can be found which contains one or more planar defects, like the one shown in Fig. 2. Here a series of planar defects, formed during particle growth, are observed which suggest a particular growth mechanism. Partially formed particles, like the Ti particle shown in Fig. 3, capture a moment in time during solidification and can give further insight into the formation process.

The $\mathrm{ZnS}$ nanowire in Fig. 4A was produced through a VLS growth technique. Semiconductor nanowires have become the subject of growing interest due to their potential application in electronic devices [2], though they typically contain planar defects which are formed during the growth process. Post-growth studies of the defects in these wires indicate a two-stage growth process which results in a saw-like structure. The wire grows quickly along its length with slower growth occurring in the perpendicular directions along only one edge as dictated by the polar nature of $\mathrm{ZnS}$. The stacking fault in Fig. 4B formed during growth, causing a c/2 translation at the interface between two "teeth".

The observation of nanoparticles with defects is instrumental in understanding how the nanoparticles form and how to tailor the production process to best suit society's growing demands. This work illustrates the significance of microscopy studies for the investigating particle formation and growth.

References

[1] N. P. Rao et al., J. Aerosol Sci. 29, (1998) 707.

[2] T. W. Odom, et al., Talanta, 67 (2005) 507. 
[3] This work is funded through NSF grant number CMS0322436 and the IGERT-DGE0114372. The authors would like to thank Prof. Steven Girshick for providing the Si and Ti particles and Dr. Subhadra Chaudhuri for the $\mathrm{ZnS}$ nanostuctures.
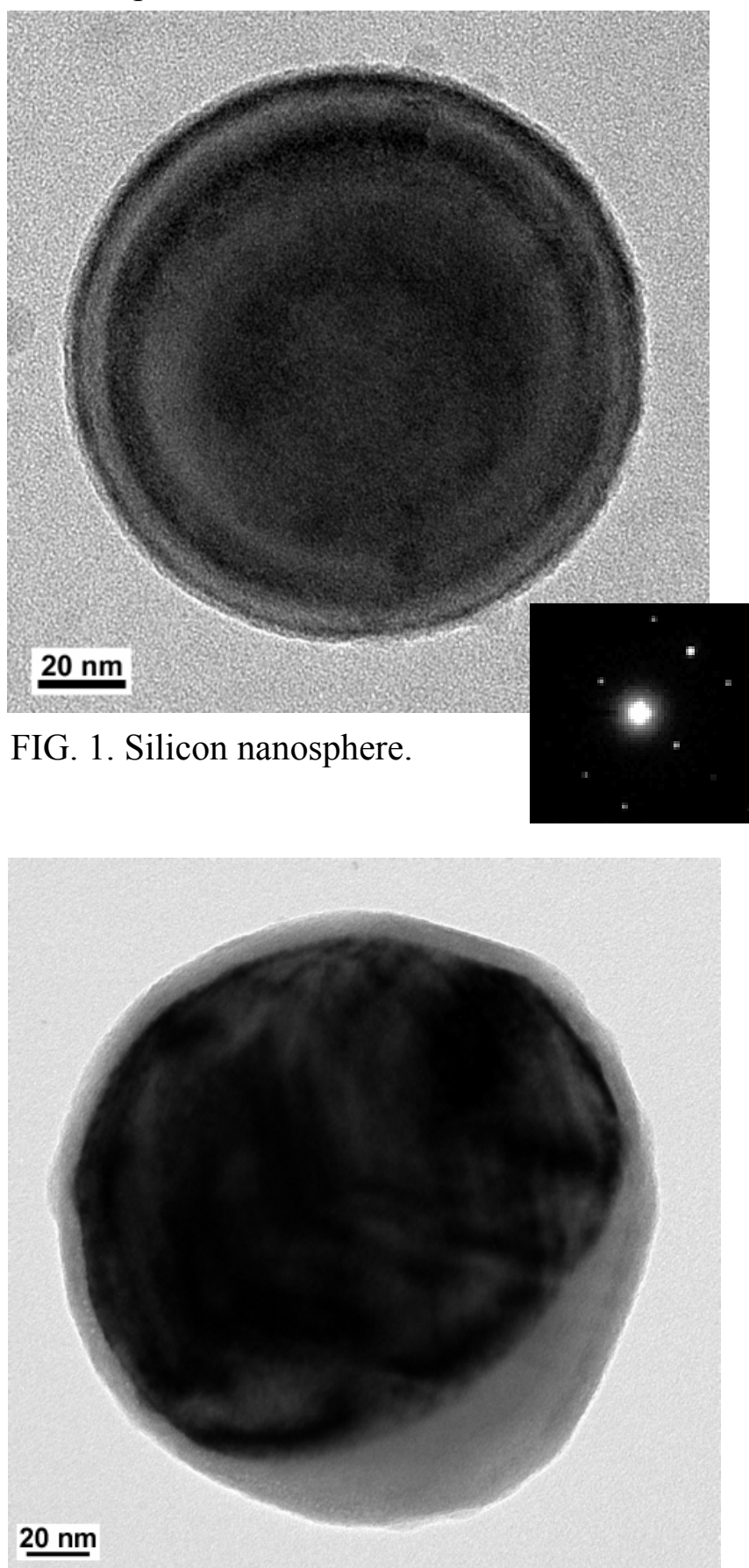

FIG. 3. (above) Partially crystallized titanium nanoparticle.

FIG. 4. (right) A) ZnS nanowire. B) Boundary formed by two growing nanosaw "teeth".

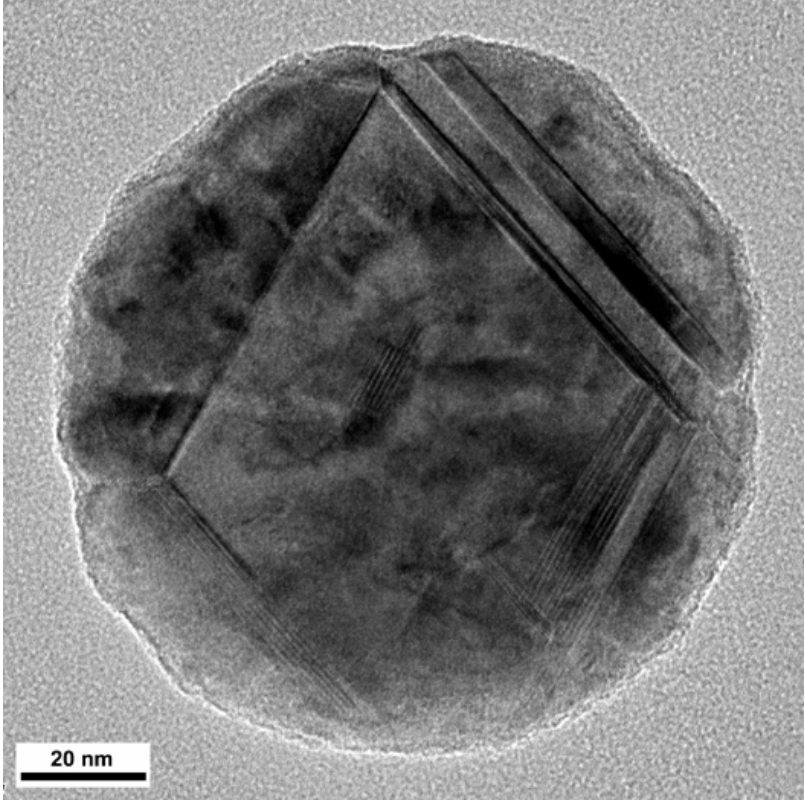

FIG. 2. Silicon "nanosphere" with multiple planar defects.
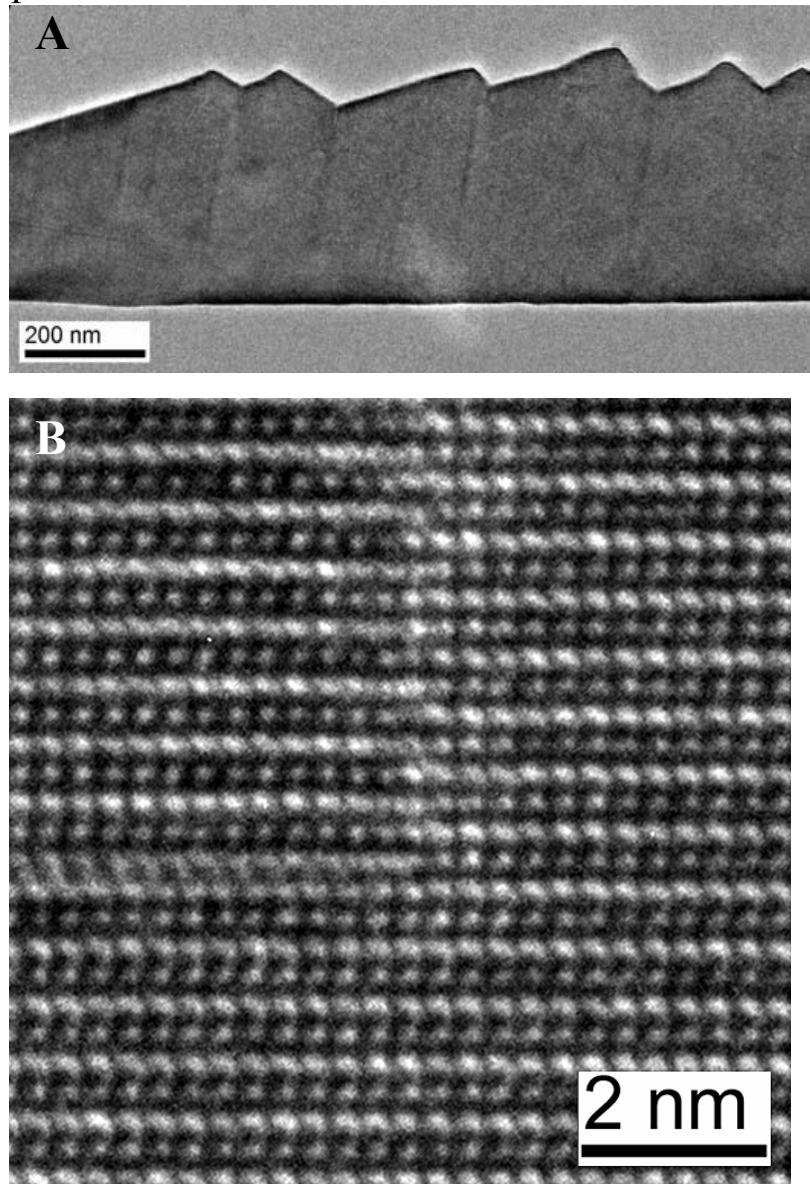